\title{
A RECONSTRUCTION ALGORITHM FOR ULTRASOUND-MODULATED DIFFUSE OPTICAL TOMOGRAPHY
}

\author{
HABIB AMMARI, EMMANUEL BOSSY, JOSSELIN GARNIER, LOC HOANG NGUYEN, \\ AND LAURENT SEPPECHER
}

(Communicated by Walter Craig)

\begin{abstract}
The aim of this paper is to develop an efficient reconstruction algorithm for ultrasound-modulated diffuse optical tomography. In diffuse optical imaging, the resolution is in general low. By mechanically perturbing the medium, we show that it is possible to achieve a significant resolution enhancement. When a spherical acoustic wave is propagating inside the medium, the optical parameter of the medium is perturbed. Using cross-correlations of the boundary measurements of the intensity of the light propagating in the perturbed medium and in the unperturbed one, we provide an iterative algorithm for reconstructing the optical absorption coefficient. Using a spherical Radon transform inversion, we first establish an equation that the optical absorption satisfies. This equation together with the diffusion model constitutes a nonlinear system. Then, solving iteratively such a nonlinear coupled system, we obtain the true absorption parameter. We prove the convergence of the algorithm and present numerical results to illustrate its resolution and stability performances.
\end{abstract}

\section{INTRODUCTION}

Diffuse optical tomography is an emerging biomedical modality that uses diffuse light to probe structural variations in the optical properties of tissue [6]. The associated inverse problem for diffuse waves consists of recovering the absorption properties of a medium of interest from boundary measurements of the light intensity. The most important current applications of diffuse optical imaging are detecting tumors in the breast and brain imaging 8 .

Let $\Omega$ be a smooth bounded domain of $\mathbb{R}^{d}$, for $d=2,3$, satisfying the interior ball condition. Let $\nu$ denote the unit normal outward vector on $\partial \Omega$ and let $\partial_{\nu}$ denote the normal derivative at $\partial \Omega$. When a laser beam is applied at a point $\mathbf{x}_{0}$ on $\partial \Omega$, the energy density $\varphi_{*}$ is governed by the diffusion equation

$$
\left\{\begin{array}{rll}
-\Delta \varphi_{*}+q_{*} \varphi_{*} & =0 & \text { in } \Omega \\
l \partial_{\nu} \varphi_{*}+\varphi_{*} & =g & \text { on } \partial \Omega
\end{array}\right.
$$

where $q_{*}$ is the (spatially-varying) optical absorption coefficient and $g \geq 0$ is a smooth approximation of the Dirac function at $\mathbf{x}_{0}$. Note that in the Robin type

Received by the editors February 8, 2012 and, in revised form, September 30, 2012.

2010 Mathematics Subject Classification. Primary 65R32, 44A12, 31B20.

Key words and phrases. Ultrasound-modulated optical imaging, diffuse optical tomography, spherical Radon transform, Helmholtz decomposition, reconstruction, convergence.

This work was supported by the ERC Advanced Grant Project MULTIMOD-267184. 
boundary condition in (1.1),$l$ is the extrapolation length 24. Throughout this paper we will assume without loss of generality that $l=1$.

The aim of this paper is to model a new method for reconstructing the optical absorption coefficient $q_{*}$ of a medium occupied by $\Omega$ from boundary measurements of $\partial_{\nu} \varphi_{*}$ on the boundary $\partial \Omega$ using only one $g$. We provide a mathematical and numerical framework for it.

We assume that the Born assumption holds, i.e., the optical coefficient $q_{*}$ takes the form

$$
q_{*}=q_{0}\left(1+\delta s_{*}\right) .
$$

Here $\delta$ is a small constant and $s_{*}$ is a smooth function whose support $D$ is known and compactly contained in the background medium $\Omega$. The Born approximation and its higher order corrections have been widely used; see, for instance, [14, 16, 21,22].

Under the Born assumption, it is known that by using boundary measurements of the normal derivative of the solution to the diffusion equation (1.1) corresponding to many $g$, it is possible to determine the optical coefficient $q_{*}$ [6, 26]. Direct reconstruction methods have been designed in this linearized case for particular experimental geometries [26]. However, the resolution of the reconstruction is usually low due to the inherent severely ill-posed character of diffuse optical imaging.

In this paper we propose a new method for reconstructing the optical coefficient, which is based on the use of mechanical perturbations of the medium. Only one $g$ is used. The idea behind our new method is to perturb the medium by a propagating acoustic wave while taking the boundary measurements of the normal derivative of the energy density corresponding to $g$. Let $\mathbf{y} \in \Omega \backslash \bar{D}$. Consider a displacement field $\mathbf{u}_{\mathbf{y}}$ generated at the source point $\mathbf{y}$ such that its support is a thin spherical shell growing at a constant speed $c$. Denote $q_{*}\left(\mathbf{x}+\mathbf{u}_{\mathbf{y}}(\mathbf{x}, t)\right)$ by $q_{u}(\mathbf{x}, \mathbf{y}, t), \mathbf{x} \in \Omega$, $t>0$, and the corresponding energy density by $\varphi_{u}(\mathbf{x}, \mathbf{y}, t)$. The presence of the propagating acoustic wave generated at the source point $\mathbf{y}$ changes the medium and yields the perturbed diffusion equation

$$
\left\{\begin{array}{rll}
-\Delta \varphi_{u}+q_{u} \varphi_{u} & =0 & \text { in } \Omega \\
\partial_{\nu} \varphi_{u}+\varphi_{u} & =g & \text { on } \partial \Omega .
\end{array}\right.
$$

Then, in order to reconstruct $q_{*}$, we cross-correlate the boundary values of the intensity of the light propagating in the medium changed by the propagation of the acoustic wave and those corresponding to the unperturbed one. We compute the quantity

$$
\int_{\partial \Omega} g\left(\partial_{\nu} \varphi_{*}-\partial_{\nu} \varphi_{u}\right) d \sigma
$$

Assume that $\mathbf{y}$ moves along a circle or a sphere. Then the use of a Helmholtz decomposition yields (3.6) for $q_{*}$ where the source term is obtained from the data given by (1.4) by using a circular or a spherical Radon transform inversion. Hence the functions $q_{*}$ and $\varphi_{*}$ satisfy the coupled system of equations (1.1) and (3.6). This nonlinear coupling suggests the iterative algorithm (3.10) -3.11) for reconstructing $q_{*}$. One of the main contributions of this paper is to prove the convergence of the iterative scheme to the true image of $q_{*}$ using the contraction fixed point theorem and to illustrate it numerically. Moreover, the high resolution and the good stability properties of the reconstructed images are shown.

The idea of mechanically perturbing the medium was first introduced in 3 . for electromagnetic imaging in free space. In [3], since the amplitude of the background 
solution is constant, an inversion of the spherical Radon transform yields the correct image. The algorithm proposed in [3] is a direct algorithm. On the other hand, it is also worth emphasizing that this approach is different from the imaging by controlled perturbations [1, 2, 5, 10, where local changes of the parameters of the medium are produced by focalizing an ultrasound beam. Both techniques lead to resolution enhancements. In imaging by controlled perturbations, the resolution is of order the size of the focal spot, while here it is of order the width of the wave front of the wave propagating in the medium. Our new method is called ultrasoundmodulated optical tomography. It significantly differs from those proposed in 9, 11,28, where the spatial scales are much smaller than those considered here. In [7], an ultrasound-modulated approach for diffuse optical imaging has been proposed. However, the modulation of the medium is different. For the acoustic wave, plane waves are used in [7] instead of spherical waves used here. This leads to a completely different model: a nonlinear partial differential equation (called zero-Laplacian) for the energy density. While the algorithms developed in [2, 10] can be used to efficiently solve such a nonlinear PDE, new techniques have to be designed for the present spherically modulated model.

The paper is organized as follows. In Section 2 we introduce some preliminary results. In Section 3 we present our reconstruction algorithm. In Section 4 we provide a proof of convergence of the algorithm. In Section 5 we illustrate the performance of the proposed algorithm in terms of resolution and stability. The paper ends with a short discussion. Throughout the paper, $C$ is a universal constant depending only on known quantities and functions.

\section{Preliminaries}

In this section, we first recall two consequences of well-known regularity results. The propositions we present here are special cases in those papers and enough for us to study ultrasound-modulated optical tomography. The reader can find the following result in [17, 19.

Proposition 2.1. Suppose that $\Omega$ is smooth. If $p \in L^{\infty}(\Omega)$, then any weak and bounded solution $\varphi$ of the equation

$$
-\Delta \varphi+p \varphi=0, \quad \text { with }\|\varphi\|_{L^{\infty}(\Omega)} \leq M,
$$

is in $\mathcal{C}^{1}(\Omega)$ and

$$
\|\varphi\|_{\mathcal{C}^{1}\left(\bar{\Omega}^{\prime}\right)} \leq c_{1}\left(M,\|p\|_{L^{\infty}(\Omega)}, \operatorname{dist}\left(\Omega^{\prime}, \partial \Omega\right)\right)
$$

for all $\Omega^{\prime} \Subset \Omega$.

The following proposition is from [12,18].

Proposition 2.2. Let $D$ be a bounded smooth domain and $\lambda<\Lambda, M$ be positive constants. If $\varphi \in L^{\infty}(D)$ is such that

$$
0<\lambda \leq \varphi \leq \Lambda \quad \text { in } D
$$

and if $f \in L^{\infty}(D)$ is such that $\|f\|_{L^{\infty}(D)} \leq M$, then the solution $q$ of

$$
\left\{\begin{aligned}
\nabla \cdot\left(\varphi^{2} \nabla q\right) & =f \quad \text { in } D \\
q & =0 \quad \text { on } \partial D
\end{aligned}\right.
$$

is in $\mathcal{C}^{1}(\bar{D})$ with

$$
\|q\|_{\mathcal{C}^{1}(\bar{D})} \leq c_{2}(\lambda, \Lambda, M)
$$


Remark 2.3. Assume that the constant $c_{2}$ in (2.3) is optimal; i.e., $c_{2}$ is the infimum of all of its possible values. Then,

$$
c_{2}(\lambda, \Lambda, \delta M) \leq \delta c_{2}(\lambda, \Lambda, M)
$$

for all $0<\delta<1$. This can be seen by multiplying both sides of (2.2) by $\delta$.

We next establish the weak comparison principle and the strong maximum principle for Laplace equations with the Robin boundary condition under consideration. The main idea of the proof is based on the weak comparison principle in 20 for Dirichlet boundary conditions, with some suitable modifications. Although this might be a well-known result, we provide a proof here for the sake of completeness. Also, our strong maximum principle is somewhat different from the classical one.

Proposition 2.4 (Weak comparison principle). Let $p$ be a nonnegative measurable function and assume that $\varphi \in H^{1}(\Omega)$ satisfies

$$
\left\{\begin{aligned}
-\Delta \varphi+p \varphi & \geq 0 \quad \text { in } \Omega \\
\partial_{\nu} \varphi+\varphi & \geq 0 \quad \text { on } \partial \Omega .
\end{aligned}\right.
$$

We have $\varphi \geq 0$ a.e. in $\Omega$.

Proof. Using $\varphi^{-}=\max \{0,-\varphi\} \geq 0$ as a test function in the variational formulation of (2.5) gives

$$
\begin{aligned}
0 & \leq \int_{\Omega} \nabla \varphi \cdot \nabla \varphi^{-} d \mathbf{x}-\int_{\partial \Omega} \partial_{\nu} \varphi \varphi^{-} d \sigma+\int_{\Omega} p \varphi \varphi^{-} d \mathbf{x} \\
& \leq \int_{\Omega} \nabla \varphi \cdot \nabla \varphi^{-} d \mathbf{x}+\int_{\partial \Omega} \varphi \varphi^{-} d \sigma+\int_{\Omega} p \varphi \varphi^{-} d \mathbf{x} \\
& =-\int_{\Omega}\left|\nabla \varphi^{-}\right|^{2} d \mathbf{x}-\int_{\partial \Omega}\left|\varphi^{-}\right|^{2} d \sigma-\int_{\Omega} p\left|\varphi^{-}\right|^{2} d \mathbf{x} .
\end{aligned}
$$

It follows that $\varphi^{-}=0$. Note that $\varphi^{-}$is admissible to be a test function because it belongs to $H^{1}(\Omega)$ (see [13]).

Proposition 2.5 (Strong maximum principle). Let $g \not \equiv 0$ be a nonnegative smooth function defined on $\partial \Omega$. Let $D \Subset \Omega$ be smooth. For all $c>0$, the solution $\varphi_{c}$ of

$$
\left\{\begin{array}{rll}
-\Delta \varphi_{c}+c \varphi_{c} & =0 & \text { in } \Omega \\
\partial_{\nu} \varphi_{c}+\varphi_{c} & =g & \text { on } \partial \Omega
\end{array}\right.
$$

is bounded and positive in D.

We need the following lemma to prove this strong maximum principle [23].

Lemma 2.6 (Hopf lemma). Let $\varphi \in \mathcal{C}^{1}(\bar{\Omega}) \cap \mathcal{C}^{2}(\Omega)$ satisfy

$$
-\Delta \varphi+c \varphi \geq 0
$$

on $\Omega$ where $c$ is a nonnegative constant. If there exists $\mathbf{x}_{0} \in \partial \Omega$ such that $\varphi\left(\mathbf{x}_{0}\right) \leq 0$ and $\varphi(\mathbf{x})>\varphi\left(\mathbf{x}_{0}\right)$ for all $\mathbf{x} \in \Omega$, then

$$
\partial_{\nu} \varphi\left(\mathbf{x}_{0}\right)<0 .
$$

Proof of Proposition 2.5. Since $g$ is nonnegative, so is $\varphi_{c}$ because of Proposition 2.4 . On the other hand, applying Proposition 2.4 again for $\|g\|_{L^{\infty}(\partial \Omega)}-\varphi$, we can see that $\varphi \leq\|g\|_{L^{\infty}(\partial \Omega)}$. The boundedness of $\varphi_{c}$ in $\Omega$, and hence $D$, has been verified. 
In order to use the Hopf lemma, we show that $\varphi_{c} \in C^{2}(\Omega)$. In fact, for all $x_{0} \in \Omega$, let $D_{1}$ and $D_{2}$ satisfying

$$
D_{1} \Subset D_{2} \Subset \Omega
$$

be two open neighbourhoods of $x_{0}$. The boundedness of $\varphi_{c}$ in the previous paragraph and Proposition 2.1 imply that $\varphi_{c}$ belongs to $C^{1}\left(\bar{D}_{2}\right)$. On the other hand, Theorem 8.8 in [13] helps us to see that $\varphi_{c} \in H^{2}\left(D_{2}\right)$. Hence, $\partial_{x_{i}} \varphi_{c}, i=1, \cdots, d$, is in $H^{1}\left(D_{2}\right)$. It also satisfies the equation

$$
-\Delta \partial_{x_{i}} \varphi_{c}+c \partial_{x_{i}} \varphi_{c}=0 .
$$

Hence, $\partial_{x_{i}} \varphi_{c}$ belongs to $C^{1}\left(\bar{D}_{1}\right)$ by Proposition 2.1. In other words, $\varphi_{c} \in C^{2}\left(\bar{D}_{1}\right)$.

We claim that $\varphi_{c}>0$ not only in $\bar{D}$ but also in $\Omega$. Assume that $\varphi_{c}\left(\mathbf{x}_{0}\right)=0$ for some $\mathbf{x}_{0} \in \Omega$. Since $g$ is not identically zero, neither is $\varphi_{c}$. Hence, we can find a point $\mathbf{x}_{1} \in \Omega$ such that $\varphi_{c}\left(\mathbf{x}_{1}\right)>0$. Without loss of generality, we can suppose that $B\left(\mathbf{x}_{1}, r\right) \subset \Omega$ with $r=\left|\mathbf{x}_{1}-\mathbf{x}_{0}\right|$ and $\varphi_{c}(\mathbf{x})>0$ for all $\mathbf{x} \in B\left(\mathbf{x}_{1}, r\right)$. Since $\varphi_{c} \in \mathcal{C}^{2}(\Omega), \varphi_{c}$ belongs to $\mathcal{C}^{1}\left(\overline{B\left(\mathbf{x}_{1}, r\right)}\right) \cap \mathcal{C}^{2}\left(B\left(\mathbf{x}_{1}, r\right)\right)$. We can apply the Hopf lemma for $\varphi_{c}$ in $B\left(\mathbf{x}_{1}, r\right)$ to get

$$
\nabla \varphi_{c}\left(\mathbf{x}_{0}\right) \cdot\left(\mathbf{x}_{1}-\mathbf{x}_{0}\right)<0 .
$$

This is a contradiction because $\varphi_{c}$ attains its minimum value at $\mathbf{x}_{0}$ and $\nabla \varphi_{c}\left(\mathbf{x}_{0}\right)=$ 0 .

\section{A ReCOnStruCtion ALGORIthm}

In order to achieve a resolution enhancement ultrasound-modulated optical tomography can be used. Its basic principles are as follows. We generate a spherical acoustic wave inside the medium. The propagation of the acoustic wave changes the absorption parameter of the medium. During the propagation of the wave we measure the light intensity on $\partial \Omega$. The aim is now to reconstruct the optical absorption coefficient from such a set of measurements with a better resolution and stability than using pure optical tomography.

In 3 , we have shown that the displacement function $\mathbf{u}$ at $\mathbf{x}$ caused by a short diverging spherical acoustic wave generated at $\mathbf{y} \in \mathbb{R}^{d} \backslash \bar{D}$ is of the form

$$
\mathbf{u}(\mathbf{x})=\mathbf{u}_{\mathbf{y}}(\mathbf{x}, t)=-\frac{\eta}{c t} w\left(\frac{|\mathbf{x}-\mathbf{y}|-c t}{\eta}\right) \frac{\mathbf{x}-\mathbf{y}}{|\mathbf{x}-\mathbf{y}|}, \quad \mathbf{x} \in \Omega,
$$

where the constant $c$ is the acoustic wave speed, $w \in \mathcal{C}^{\infty}\left(\mathbb{R}, \mathbb{R}^{+}\right.$) (called the shape function) is with support contained in $[-1,1], \eta$ is a positive parameter representing the thickness of the wavefront, i.e., the thickness of the support of the displacement field $\mathbf{u}$, and $t$ is understood as the time parameter.

Now, from (1.1) and (1.3), it follows that

$$
\left\|\varphi_{u}-\varphi_{*}\right\|_{H^{1}(\Omega)} \leq c\left\|q_{u}-q_{*}\right\|_{L^{2}(\Omega)},
$$

for some positive constant $c$, and therefore [3]

$$
\int_{\partial \Omega} g\left(\partial_{\nu} \varphi_{*}-\partial_{\nu} \varphi_{u}\right) d \sigma=\int_{\Omega} \varphi_{*} \varphi_{u}\left(q_{*}-q_{u}\right) d \mathbf{x} \approx-\int_{\Omega} \varphi_{*}^{2} \nabla q_{*} \cdot \mathbf{u} d \mathbf{x}
$$

Since $\partial_{\nu} \varphi_{*}$ and $\partial_{\nu} \varphi_{u}$ can be measured on $\partial \Omega$, it is possible to evaluate the quantity $\int_{\partial \Omega} g\left(\partial_{\nu} \varphi_{*}-\partial_{\nu} \varphi_{u}\right) d \sigma$ for all $\mathbf{y}, t$. This quantity is nothing other than the cross-correlations between the boundary measurements in the perturbed and unperturbed media. 
Next, from (3.2) we establish an equation for $q_{*}$. Using Helmholtz decomposition, we write

$$
\varphi_{*}^{2} \nabla q_{*}=-\nabla \psi+\nabla \times \Psi .
$$

Here, in order to insure the uniqueness of $\psi$ and $\Psi$ we assume that $\Omega$ is simply connected, $\Psi$ is such that $\nabla \cdot \Psi=0$, and we supply the boundary conditions $\partial_{\nu} \psi=-\varphi_{*}^{2} \partial_{\nu} q_{*}$ and $\Psi \times \nu=0$ on $\partial \Omega$.

Since $\mathbf{u}$ takes the radial form (3.1), integration by parts yields

$$
\int_{\Omega} \nabla \times \Psi \cdot \mathbf{u} d \mathbf{x}=0
$$

and so (3.2) can be rewritten as

$$
\int_{\partial \Omega} g\left(\partial_{\nu} \varphi_{*}-\partial_{\nu} \varphi_{u}\right) d \sigma \approx \int_{\Omega} \nabla \psi \cdot \mathbf{u} d \mathbf{x}
$$

Hence, $\psi$ can be constructed and considered as the given data by employing the spherical Radon transform, as was done in Section 5 in [3].

Let

$$
N_{u}(\mathbf{y}, r):=\int_{\partial \Omega} g(\mathbf{x})\left(\partial_{\nu} \varphi_{*}(\mathbf{x})-\partial_{\nu} \varphi_{u}(\mathbf{x}, \mathbf{y}, r / c)\right) d \sigma(\mathbf{x}),
$$

where $\mathbf{u}_{\mathbf{y}}$ is given by (3.1).

For $f \in \mathcal{C}^{0}\left(\mathbb{R}^{d}\right)$ and $E \subset \mathbb{R}^{d}$, define the spherical Radon transform of $f$ over $E$ by

$$
\mathcal{R} f(\mathbf{y}, r)=\frac{1}{|S|} \int_{S} f(\mathbf{y}+r \boldsymbol{\xi}) d \sigma(\boldsymbol{\xi}) \quad \mathbf{y} \in E, r>0,
$$

where $|S|$ is the surface of the unit sphere $S$ and $d \sigma$ is the surface measure over $S$.

From [3] the following lemma holds.

Lemma 3.1. Fix $\mathbf{y} \in \Omega \backslash \bar{D}$ and let $r_{0}>0$. Suppose that $q_{*}(\mathbf{x})=q_{0}$ for $\mathbf{x} \in$ $B\left(\mathbf{y}, r_{0}\right)$, where $B$ is the ball of center $\mathbf{y}$ and radius $r_{0}$. Suppose also that $q_{*} \in$ $\mathcal{C}^{1, \beta}(\bar{\Omega})$ and $\eta$ is small enough. Then, for all $r>r_{0}$ and $\eta \ll r$, we have

$$
\mathcal{R} \psi(\mathbf{y}, r) \approx-\frac{1}{\eta^{2}\|w\|_{L^{1}}|S|} \int_{r_{0}}^{r} \frac{N_{u}(\mathbf{y}, \rho)}{\rho^{d-2}} d \rho .
$$

Having in hand $\psi$ from the cross-correlations between boundary measurements using a spherical Radon transform inversion, we take the divergence of (3.3) to arrive at

$$
-\nabla \cdot\left(\varphi_{*}^{2} \nabla q_{*}\right)=\Delta \psi
$$

This together with (1.2) also implies that

$$
-\Delta \psi=\delta q_{0} \nabla \cdot\left(\varphi_{*}^{2} \nabla s_{*}\right),
$$

where $\delta$ and $s_{*}$ were introduced in (1.2). Assume that $q_{*}$ is bounded from below and above by two known positive constants $q$ and $\bar{q}$ respectively. Since $\varphi_{*}$ solves problem (2.1) with $q_{*}$ replacing $p$ and $\Lambda$, which will be defined later in Lemma 4.1. by replacing $M$, its $\mathcal{C}^{1}(\bar{D})$ norm is bounded. We assume further that $\delta$ is small and $s_{*}$ is smooth, with known bound on its $\mathcal{C}^{2}(D)$ norm, to guarantee that $\|\Delta \psi\|_{L^{\infty}(D)}$ 
is bounded in the order of $\delta$. The analysis allows us to recover $q_{*}$ in $D$ by solving the system of equations for the two unknowns $\varphi$ and $q$ :

$$
\left\{\begin{array}{rll}
-\Delta \varphi+q \varphi & =0 & \text { in } \Omega \\
\partial_{\nu} \varphi+\varphi & =g & \text { on } \partial \Omega
\end{array}\right.
$$

and

$$
\left\{\begin{aligned}
-\nabla \cdot\left(\varphi^{2} \nabla q\right) & =\Delta \psi & & \text { in } D \\
q & =q_{0} & & \text { on } \partial D
\end{aligned}\right.
$$

where

$$
|\Delta \psi|_{L^{\infty}(\Omega)}=O(\delta) \quad \text { as } \delta \ll 1 .
$$

This suggests the following algorithm:

(1) Define the initial guess $q^{(0)}=q_{0}$.

(2) Establish an iterating sequence $\left\{q^{(n)}\right\}$ as follows:

(a) For $n \geq 1$, solve

$$
\left\{\begin{array}{rlll}
-\Delta \varphi^{(n)}+\hat{q}^{(n-1)} \varphi^{(n)} & = & 0 & \text { in } \Omega \\
\partial_{\nu} \varphi^{(n)}+\varphi^{(n)} & = & g & \text { on } \partial \Omega
\end{array}\right.
$$

where

$$
\hat{p}:=\min \{\max \{p, \underline{q}\}, \bar{q}\} .
$$

(b) Find $q^{(n)}$ by solving

$$
\left\{\begin{aligned}
-\nabla \cdot\left(\left(\varphi^{(n)}\right)^{2} \nabla q^{(n)}\right) & =\Delta \psi & & \text { in } D, \\
q^{(n)} & =q_{0} & & \text { on } \partial D
\end{aligned}\right.
$$

and defining $q^{(n)}=q_{0}$ in $\Omega \backslash D$.

(3) The convergent function of $\left\{q^{(n)}\right\}$ is the true optical absorption coefficient $q_{*}$.

Remark 3.2. The convergence of $\left\{q^{(n)}\right\}$, mentioned in step 3 , will be shown by the Banach fixed point theorem in the next section. This also implies the well-posedness of the system constituted by (3.8) and (3.9).

Remark 3.3. Problem (3.10) is uniquely solvable because we are able to avoid the case that $\left(\varphi^{(n)}\right)^{2}$ approaches 0 or $\infty$ somewhere inside $D$ in the next section (see Lemma 4.1).

Remark 3.4. We modify $q^{(n-1)}$ by $\hat{q}^{(n-1)}$ in (3.10) because of the obvious inequality

$$
\left|\hat{p}-q_{*}\right| \leq\left|p-q_{*}\right|,
$$

which makes the proof of the algorithm easier and may increase the rate of convergence.

\section{The CONVERGEnCE OF THE ITERATIVE ALGORITHM}

Define the open set of $L^{\infty}(\Omega)$,

$$
\mathcal{Q}=\left\{p \in L^{\infty}(\Omega): \underline{q}<p<\bar{q}\right\}
$$

and the map

$$
\begin{aligned}
F_{1}: \mathcal{Q} & \rightarrow H^{1}(\Omega) \\
q & \mapsto F_{1}[q]=\varphi, \text { where } \varphi \text { is the solution of (3.8). }
\end{aligned}
$$

We have the following result. 
Lemma 4.1. For all $q \in \mathcal{Q}, F_{1}[q]$ is in $L^{\infty}(\Omega)$. There exists a positive constant $\Lambda(\underline{q}, \bar{q})$ such that

$$
\left|F_{1}[q](\mathbf{x})\right| \leq \Lambda, \quad \forall \mathbf{x} \in \Omega .
$$

Moreover, for any $D \Subset \Omega$, there exists a positive constant $\lambda(D, \underline{q}, \bar{q})$ such that

$$
\lambda \leq F_{1}[q](\mathbf{x}), \quad \forall \mathbf{x} \in D .
$$

Proof. Let $\varphi_{q}$ and $\varphi_{\bar{q}}$ be the solutions of (2.6) with $c$ replaced by $q$ and $\bar{q}$, respectively. It follows by Proposition 2.4 that

$$
\varphi_{\bar{q}} \leq \varphi \leq \varphi_{\underline{q}} \quad \text { in } \Omega .
$$

On the other hand, we can apply Proposition 2.5 to see that

$$
\varphi_{\bar{q}}>0 \quad \text { in } D \text {. }
$$

The lemma is proved by letting $\lambda=\inf _{D} \varphi_{\bar{q}}$ and $\Lambda=\sup _{\Omega} \varphi_{\underline{q}}$.

Lemma 4.2. The map $F_{1}$ is Fréchet differentiable. Its derivative at $q$ is given by

$$
D F_{1}[q](h)=\phi,
$$

for $h \in L^{\infty}(\Omega)$, where $\phi$ solves

$$
\left\{\begin{aligned}
-\Delta \phi+q \phi & =-h \varphi & & \text { in } \Omega, \\
\partial_{\nu} \phi+\phi & =0 & & \text { on } \partial \Omega,
\end{aligned}\right.
$$

with $\varphi=F_{1}[q]$. Moreover, $D F_{1}[q]$ can be continuously extended to the whole $L^{2}(\Omega)$ by the same formula in (4.6) with

$$
\left\|D F_{1}[q]\right\|_{\mathcal{L}\left(L^{2}(\Omega), H^{1}(\Omega)\right)} \leq C \Lambda,
$$

where $\Lambda$ was defined in Lemma 4.1.

Proof. Let $\varphi^{\prime}$ be the solution of (3.8) with $q+h$ replacing $q$, assuming $\|h\|_{L^{\infty}(\Omega)} \ll 1$ so that $q+h \in \mathcal{Q}$ a.e. in $\Omega$. Note that $\varphi^{\prime}-\varphi$ solves

$$
\left\{\begin{aligned}
-\Delta\left(\varphi^{\prime}-\varphi\right)+(q+h)\left(\varphi^{\prime}-\varphi\right) & =-h \varphi & & \text { in } \Omega, \\
\partial_{\nu}\left(\varphi^{\prime}-\varphi\right)+\left(\varphi^{\prime}-\varphi\right) & =0 & & \text { on } \partial \Omega .
\end{aligned}\right.
$$

Using $\varphi^{\prime}-\varphi$ as a test function in the variational formulation of the problem above gives

$$
\left\|\varphi^{\prime}-\varphi\right\|_{H^{1}(\Omega)} \leq C\|h\|_{L^{\infty}(\Omega)}\|\varphi\|_{L^{2}(\Omega)} .
$$

On the other hand, since $\varphi^{\prime}-\varphi-\phi$ solves

$$
\left\{\begin{aligned}
-\Delta\left(\varphi^{\prime}-\varphi-\phi\right)+q\left(\varphi^{\prime}-\varphi-\phi\right) & =-h\left(\varphi^{\prime}-\varphi\right) & & \text { in } \Omega, \\
\partial_{\nu}\left(\varphi^{\prime}-\varphi-\phi\right)+\left(\varphi^{\prime}-\varphi-\phi\right) & =0 & & \text { on } \partial \Omega,
\end{aligned}\right.
$$

we can apply the argument above to obtain

$$
\left\|\varphi^{\prime}-\varphi-\phi\right\|_{H^{1}(\Omega)} \leq C\|h\|_{L^{\infty}(\Omega)}\left\|\varphi^{\prime}-\varphi\right\|_{L^{2}(\Omega)} .
$$

Combining (4.8) and (4.9) shows that

$$
\left\|\varphi^{\prime}-\varphi-\phi\right\|_{H^{1}(\Omega)} \leq C\|h\|_{L^{\infty}(\Omega)}^{2}\|\varphi\|_{L^{2}(\Omega)},
$$

which implies

The first part of the lemma follows.

$$
\lim _{\|h\|_{L^{\infty}(\Omega) \rightarrow 0}} \frac{\left\|\varphi^{\prime}-\varphi-\phi\right\|_{H^{1}(\Omega)}}{\|h\|_{L^{\infty}(\Omega)}}=0 .
$$


Because of Lemma 4.1 and Proposition 2.4. which shows that $\varphi \in L^{\infty}(\Omega)$, problem (4.6) is uniquely solvable for all $h \in L^{2}(\Omega)$, and therefore the extension $D F_{1}[q]: L^{2}(\Omega) \rightarrow H^{1}(\Omega)$ is well-defined. Its continuity and (4.7) can be deduced, using $\phi$ as a test function in the variational formulation of (4.6) and applying Lemma 4.1 .

$$
\|\phi\|_{H^{1}(\Omega)} \leq C\|h\|_{L^{2}(\Omega)}\|\varphi\|_{L^{\infty}(\Omega)} .
$$

We next introduce another open set of $L^{\infty}(\Omega)$ :

$$
\mathcal{P}=\left\{\rho \in L^{\infty}(\Omega): \frac{\lambda}{2}<\rho<2 \Lambda \text { in } D\right\} .
$$

Let

$$
\begin{aligned}
F_{2}: \mathcal{P} & \rightarrow H^{1}(\Omega) \\
\varphi & \mapsto F_{2}[\varphi]=q, \text { where } q \text { is the solution of (3.9) in } D \text { and } q=q_{0} \text { on } \Omega \backslash D .
\end{aligned}
$$

The following lemma can be proved in the same manner as Lemma 4.2

Lemma 4.3. The map $F_{2}$ is Fréchet differentiable. Its derivative at $\varphi$ is given by

$$
D F_{2}[\varphi](h)=Q,
$$

for $h \in L^{\infty}(\Omega)$, where $Q$ solves

$$
\left\{\begin{aligned}
-\nabla \cdot\left(\varphi^{2} \nabla Q\right) & =\nabla \cdot(2 \varphi h \nabla q) & & \text { in } D \\
Q & =0 & & \text { on } \partial D
\end{aligned}\right.
$$

with $q=F_{2}[\varphi]$ being the solution of (3.9) and $Q=0$ in $\Omega \backslash D$. Moreover, $D F_{2}[\varphi]$ can be extended continuously to $L^{2}(\Omega)$ and

$$
\left\|D F_{2}[\varphi]\right\|_{\mathcal{L}\left(L^{2}(\Omega), H^{1}(\Omega)\right)} \leq \frac{2 \delta \Lambda q_{0}}{\lambda^{2}} c_{2}(\lambda, \Lambda, M),
$$

where $M$ is an upper bound of $\left\|\nabla \cdot\left(\varphi_{*} \nabla s_{*}\right)\right\|_{L^{\infty}(D)}$.

Proof. Since evaluating the derivative of $F_{2}$ at $\varphi$ is similar to doing so in Lemma 4.2, we only verify the well-definedness of the extension of $D F_{2}[\varphi]$ and (4.13). Since $\varphi \in \mathcal{P}$, we can apply Proposition 2.2 to see that the solution $q$ of $(\underline{3.9})$ is in $\mathcal{C}^{1}(\bar{D})$ and

$$
\left\|q-q_{0}\right\|_{\mathcal{C}^{1}(\bar{D})} \leq c_{2}(\lambda, \Lambda, \delta M)
$$

As a consequence, since $q=q_{0}$ on $\Omega \backslash D$, we deduce that

$$
\|\nabla q\|_{L^{\infty}(\Omega)} \leq c_{2}(\lambda, \Lambda, \delta M) \leq \delta q_{0} c_{2}(\lambda, \Lambda, M) .
$$

Thus, (4.12) is uniquely solvable if $h \in L^{2}(\Omega)$. This shows how to extend $D F_{2}[\varphi]$ to $L^{2}(\Omega)$.

In order to prove (4.13), we use $Q$ as a test function in the variational formulation of (4.12) and employ (4.14) to get

$$
\begin{aligned}
\lambda^{2} \int_{D}|\nabla Q|^{2} d \mathbf{x} & \leq \int_{D} \varphi^{2}|\nabla Q|^{2} d \mathbf{x} \\
& \leq 2 \Lambda\|\nabla q\|_{L^{\infty}(D)} \int_{D}|h||\nabla Q| d \mathbf{x} \\
& \leq 2 \delta \Lambda c_{2}(\lambda, \Lambda, M)\|h\|_{L^{2}(D)}\|\nabla Q\|_{L^{2}(D)}
\end{aligned}
$$


Therefore,

$$
\|Q\|_{H_{0}^{1}(D)} \leq \frac{2 \delta \Lambda q_{0}}{\lambda^{2}} c_{2}(\lambda, \Lambda, M)
$$

and the proof is complete.

Our main result in this section is the following.

Theorem 4.4. Assume that $\left\|s_{*}\right\|_{\mathcal{C}^{2}(D)} \leq M$ and $\underline{q}, \bar{q}$, and $M$ are given. If $\delta$ is sufficiently small, then the iteration sequence in the algorithm converges in $L^{2}(\Omega)$ to $q_{*}$, the unique solution of (3.8) and (3.9).

Proof. Introduce the map

$$
F[q]=F_{2} \circ F_{1}[q]
$$

defined on $\mathcal{Q}$. Thanks to (4.3) and (4.4), the range of $F_{1}$ is contained in the domain of $F_{2}$. This shows how the definition above makes sense. Considering $F$ as the map $\mathcal{P} \rightarrow L^{2}(\Omega)$, using the standard chain rule in differentiation and the fact that $H^{1}(\Omega) \subset L^{2}(\Omega)$, we have

$$
D F[q]: L^{\infty}(\Omega) \rightarrow L^{2}(\Omega)
$$

given by

$$
D F[q](h)=D F_{2}\left[F_{1}[q]\right]\left(D F_{1}[q](h)\right)
$$

is the Fréchet derivative of $F$. Moreover, by Lemmas 4.2 and 4.3, $D F[q]$ can be extended continuously to $L^{2}(\Omega)$ with

$$
\|D F[q]\|_{\mathcal{L}\left(L^{2}(\Omega), L^{2}(\Omega)\right)} \leq\left\|D F_{1}[q]\right\|_{\mathcal{L}\left(L^{2}(\Omega), H^{1}(\Omega)\right)}\left\|D F_{2}[q]\right\|_{\mathcal{L}\left(L^{2}(\Omega), H^{1}(\Omega)\right)} \leq C \delta .
$$

Recall from the algorithm that $q^{(0)}=q_{0}$ is the initial guess for the true coefficient $q_{*}$, and for $n \geq 1$, define

$$
q^{(n)}=F\left[T q^{(n-1)}\right] \quad n \geq 1,
$$

where $T(p)=\min \{\max \{p, \underline{q}\}, \bar{q}\}$. Note that for all $m, n \geq 1$,

$$
\begin{aligned}
\left\|F\left[T q^{(n)}\right]-F\left[T q^{(m)}\right]\right\|_{L^{2}(\Omega)} & =\left\|\int_{0}^{1} D F\left[(1-t) T q^{(n)}+t T q^{(m)}\right]\left(q^{(m)}-q^{(n)}\right) d t\right\|_{L^{2}(\Omega)} \\
& \leq C \delta\left\|q^{(m)}-q^{(n)}\right\|_{L^{2}(\Omega)} .
\end{aligned}
$$

Thus, if $\delta$ is small enough, then

$$
F \circ T: L^{2}(\Omega) \rightarrow L^{2}(\Omega)
$$

is a contraction map. Let $q^{*}$ denote the fixed point of $F \circ T$ and hence the convergent point of $q^{(n)}$. Since $q_{*}$, the true absorption coefficient, is a fixed point of $F$ and is in the interval $[q, \bar{q}]$, it is the fixed point of $F \circ T$. Therefore, $q^{*}=q_{*}$ and the proof is complete.

Remark 4.5. Let $\Omega_{i}$ be the support of $q_{*}-q_{0}$. It is easy to see that the result of this paper holds when $\delta\left|\Omega_{i}\right| \ll|\Omega|$. In fact, if the support of $q_{*}-q_{0}$ is small, then $\delta$ can be taken quite large. 


\section{Numerical experiments}

As a test case, we consider $\Omega=(-1,1)^{2}$ and $q_{0}=1$. We set

$$
q_{*}(\mathbf{x})=1+\left(q_{i}-1\right) \mathbf{1}_{\Omega_{i}}(\mathbf{x}),
$$

with $\Omega_{i} \Subset \Omega$ and $q_{i}>1$ a constant. Here, $\mathbf{1}_{\Omega_{i}}$ denotes the characteristic function of $\Omega_{i}$. We take the dimensionless shape function $w$ in (3.1) as follows:

$$
w(\alpha)=e^{1 /\left(\alpha^{2}-1\right)}, \quad \alpha \in[-1,1] .
$$

We generate the cross-correlation between boundary measurements $N_{u}$ given by (3.4), with $\mathbf{u}=\mathbf{u}_{\mathbf{y}}$ for sampling points $\mathbf{y}$ ( $\operatorname{such}$ that $q_{*}(\mathbf{y})=q_{0}$ ) on the unit circle and sampling radii $r \in(0,2)$. Then, using Lemma 3.1 and adopting the same numerical approach as in [4, we generate the data $\Psi$ by inverting the spherical Radon transform. In the case where the number of sampling points $\mathbf{y}$ is small, the total variation regularization method developed in [4. can be used. Problems (3.10) and (3.11) are solved iteratively using a finite element code. We use a structured mesh with $10^{4}$ vertices and $P 1$ finite elements.

In order to measure the quality of the reconstruction, we introduce, as in [3], two indicators of the errors made in the image. Let $m=\min \left(q_{\mathrm{mes}}\right)$ and $M=\max \left(q_{\mathrm{mes}}\right)$. We compute the support of $q_{\text {mes }}-q_{0}$ by

$$
\Omega_{i, \mathrm{mes}}=\left\{q_{\mathrm{mes}}-1>\frac{M-m}{2}\right\}
$$

and define a position error by

$$
E_{\mathrm{pos}}=\frac{\left|\Omega_{i} \triangle \Omega_{i, \mathrm{mes}}\right|}{2\left|\Omega_{i}\right|}
$$

with $\Omega_{i}$ being the correct support of $q_{*}-q_{0}$. Here, $\left|\Omega_{i} \triangle \Omega_{i, \text { mes }}\right|$ denotes the symmetric difference between $\Omega_{i}$ and $\Omega_{i, \text { mes }}$. The second quantity we have to recover is $q_{i}$, the correct value of $q_{*}$ in the inclusion. For doing so, let us define an estimation of $q_{i}$ by

$$
q_{i, \mathrm{mes}}=\frac{1}{\left|\Omega_{i, \mathrm{mes}}\right|} \int_{\Omega_{i, \mathrm{mes}}} q_{\mathrm{mes}}
$$

and introduce the relative error for this estimation as follows:

$$
E_{\mathrm{val}}=\frac{\left|q_{i, \mathrm{mes}}-q_{i}\right|}{\left|q_{i}-1\right|}
$$

Finally, we introduce the relative $L^{2}$-error,

$$
\left\|\varphi^{(n)}-\varphi_{*}\right\|_{L^{2}(\Omega)} /\left\|\varphi_{*}\right\|_{L^{2}(\Omega)},
$$

where $n$ is the number of iterations and $\varphi_{*}$ is the true energy density.

As illustrated in Figure1, if $\left|q_{i}-1\right|\left|\Omega_{i}\right| \ll|\Omega|$, then one iteration could be enough to obtain a quite resolved image (i.e., with high resolution) since the relative $L^{2}$ error $\left\|\varphi^{(1)}-\varphi_{*}\right\|_{L^{2}(\Omega)} /\left\|\varphi_{*}\right\|_{L^{2}(\Omega)}$ is very small (of order $10^{-10}$ in the example in Figure 10.

In Figure 2 we consider the same example as in Figure 1. We plot the behaviors of $E_{\mathrm{val}}$ and $E_{\mathrm{pos}}$ as functions of $\eta$. It can be seen that the smaller $\eta$ is, the better the reconstruction. However, there is a saturation effect for very small $\eta$ due to the finite element discretization. 


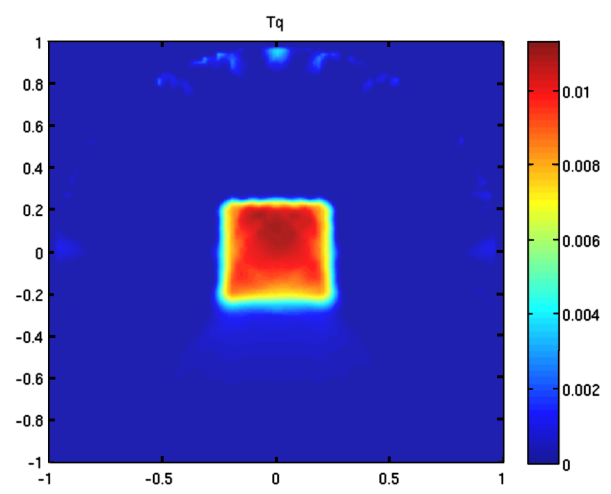

FIgURE 1. Reconstruction after one iteration with $\eta=0.02, q_{i}=$ 1.01, $\Omega_{i}=(-0.25,0.25)^{2}$, and the measurements $N_{u}$ are for 50 sources on the unit circle and for $r \in(0,2)$.

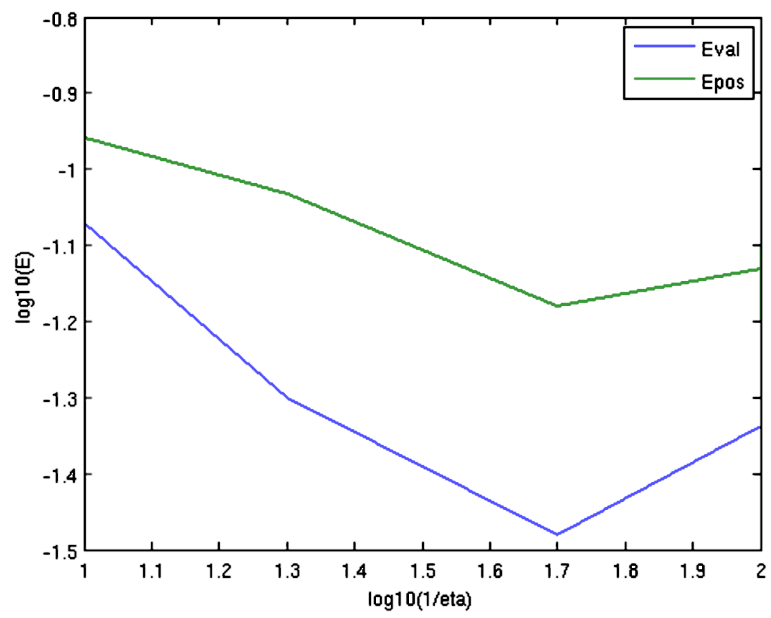

FiguRE 2. Reconstruction errors $E_{\text {val }}$ and $E_{\text {pos }}$ as functions of $\eta$.

Next, we show in Figure 3 that a few iterations are necessary to reconstruct a resolved image if $\left|q_{i}-1\right|\left|\Omega_{i}\right| /|\Omega|$ is not too small. In Figure 3, the reconstructed images after one, two, and three iterations are given. In Figure 4 it can be seen that while the support of the inclusion is quite well-reconstructed at the first iteration (it is in fact the support of the data $\Psi$ ), a few iterations are needed in order to find a good approximation of the value of the optical absorption parameter.

Finally, we illustrate the stability of the proposed algorithm. For doing so, we add to the measurements a Gaussian white noise with standard deviation ranging from $0 \%$ to $10 \%$ of the $L^{\infty}$ norm of $N_{u}$ and compute the root mean square errors of the optical absorption parameter, $\mathbb{E}\left(E_{\text {val }}^{2}\right)^{1 / 2}$, and the position, $\mathbb{E}\left(E_{\text {pos }}^{2}\right)^{1 / 2}$, as functions of the noise level. Here $\mathbb{E}$ stands for the expectation (mean value). In Figure 5 , 

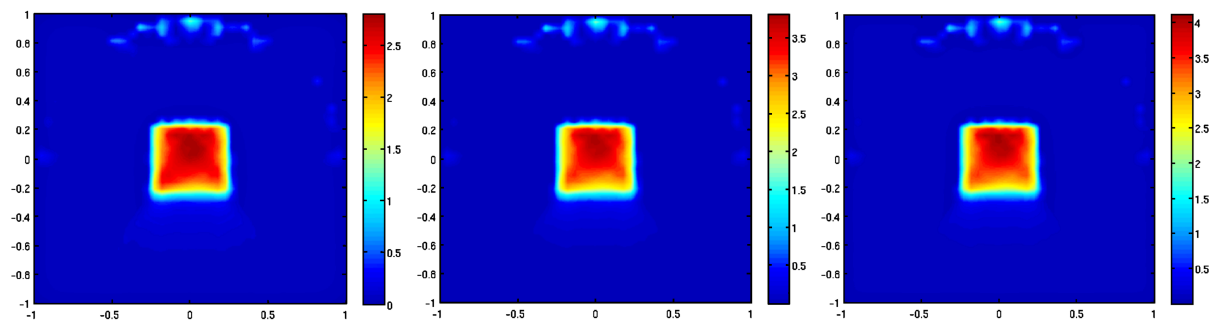

Figure 3. Reconstruction after (from left to right) one, two, and three iterations with $\eta=0.02, q_{i}=3$ and $\Omega_{i}=(-0.25,0.25)^{2}$, and the measurements $N_{u}$ are for 50 sources on the unit circle and for $r \in(0,2)$.
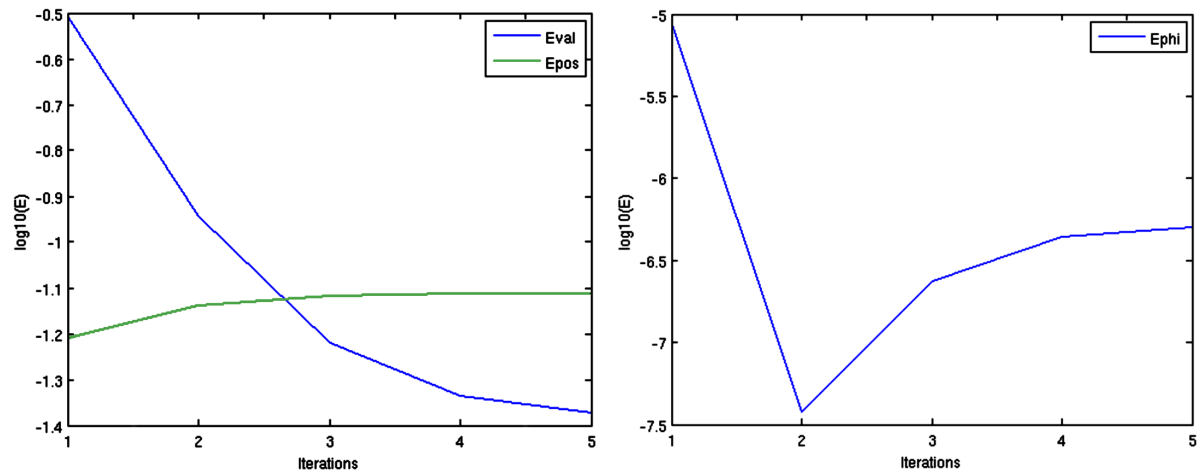

FIguRE 4. Left: Reconstruction errors $E_{\text {val }}$ and $E_{\text {pos }}$ as functions of the number of iterations. Right: Relative $L^{2}$-error on $\varphi^{(n)}$, where $n$ is the number of iterations.

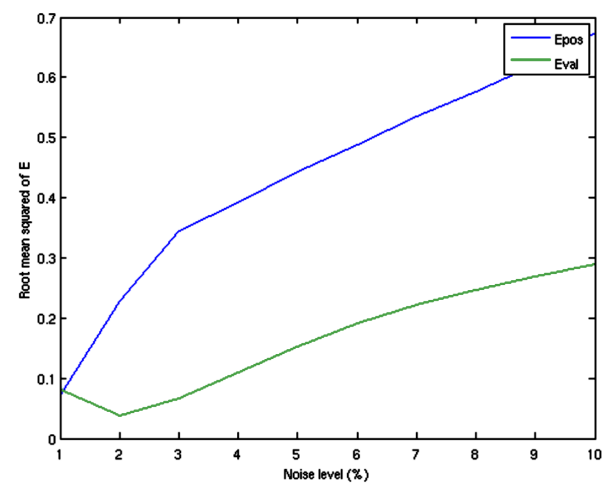

FiguRE 5. Root mean square errors of the position and the value of $q_{\text {meas }}$ for a noise level from $0 \%$ to $10 \%$. 
we compute 100 realizations of the measurement noise and apply our algorithm for estimating both the shape and the optical absorption of the inclusion. Figure 5 gives, for $\eta=0.02, \mathbb{E}\left(E_{\text {val }}^{2}\right)^{1 / 2}$ and $\mathbb{E}\left(E_{\text {pos }}^{2}\right)^{1 / 2}$ as functions of the noise level. It shows the robustness of the proposed approach. It also shows that finding the value of the optical absorption parameter is more stable than locating the inclusion. This seems to be due to the diffusion character of the problem satisfied by the optical absorption distribution.

\section{Conclusion}

In this paper we have presented a new algorithm for ultrasound-modulated optical diffuse tomography. The modulation of light is due to the propagation of spherical acoustic waves. It leads to a coupled system of equations. Iteratively solving such a system yields a resolved image for the optical absorption coefficient under the Born approximation. The algorithm has good stability properties. Its performance depends on the boundary data. In order to obtain optimal images in the sense of resolution and stability, the boundary data has to be chosen in such a way that the interior of the domain is illuminated. In the case when the Born approximation is not valid, an optimal control approach can be designed and its convergence can be proved if the initial guess is close enough to the true solution. In a forthcoming work, we will present such an approach and quantify its sensitivity to measurement noise and its resolution as a function of the signal-to-noise ratio.

The result of this paper can be extended to acousto-electromagnetic tomography developed in [3]. In [3], the background solution, being a plane wave, has constant amplitude. Even though there is no maximum principle for the Helmholtz equation, the present approach applies to acousto-electromagnetic tomography if one can explicitly check that the amplitude of the background solution has a positive lower bound in the domain. This is the case, for example, when the background solution is a spherical wave emitted at a point outside the domain or in its boundary.

\section{REFERENCES}

[1] Habib Ammari, An introduction to mathematics of emerging biomedical imaging, Mathématiques \& Applications (Berlin) [Mathematics \& Applications], vol. 62, Springer, Berlin, 2008. MR2440857 (2010j:44002)

[2] H. Ammari, E. Bonnetier, Y. Capdeboscq, M. Tanter, and M. Fink, Electrical impedance tomography by elastic deformation, SIAM J. Appl. Math. 68 (2008), no. 6, 1557-1573, DOI 10.1137/070686408. MR2424952 (2009h:35439)

[3] H. Ammari, E. Bossy, J. Garnier, and L. Seppecher, Acousto-electromagnetic tomography, SIAM J. Appl. Math., 72 (2012), pp. 1592-1617. MR3022278

[4] H. Ammari, E. Bretin, V. Jugnon, and A. Wahab, Photoacoustic imaging for attenuating acoustic media, Lecture Notes in Math., vol. 2035, 57-84, Springer-Verlag, Berlin, 2011. MR 3024670

[5] Habib Ammari, Yves Capdeboscq, Frédéric de Gournay, Anna Rozanova-Pierrat, and Faouzi Triki, Microwave imaging by elastic deformation, SIAM J. Appl. Math. 71 (2011), no. 6, 2112-2130, DOI 10.1137/110828241. MR2873260

[6] S. R. Arridge, Optical tomography in medical imaging, Inverse Problems 15 (1999), no. 2, R41-R93, DOI 10.1088/0266-5611/15/2/022. MR.1684463 (2000b:78023)

[7] G. Bal and J. C. Schotland, Inverse scattering and acousto-optic imaging, Phys. Rev. Lett. 104 (2010), 043902. 
[8] D. A. Boas, D. H. Brooks, E. L. Miller, C. A. DiMarzio, M. Kilmer, R. J. Gaudette, and Quan Zhang, Imaging the body with diffuse optical tomography, Signal Processing Magazine, IEEE 18 (2001), 57-75.

[9] E. Bossy, A. R. Funke, K. Daoudi, A. C. Boccara, M. Tanter, and M. Fink, Transient optoelastography in optically diffusive media, Appl. Phys. Lett. 90 (2007), 174111.

[10] Y. Capdeboscq, J. Fehrenbach, F. de Gournay, and O. Kavian, Imaging by modification: numerical reconstruction of local conductivities from corresponding power density measurements, SIAM J. Imaging Sci. 2 (2009), no. 4, 1003-1030, DOI 10.1137/080723521. MR2559157(2011c:35611)

[11] D. S. Elson, R. Li, C. Dunsby, R. Eckersley, and M. X. Tang, Ultrasound-mediated optical tomography: a review of current methods, Interface Focus, DOI 10.1098/rsfs.2011.0021.

[12] M. Giaquinta and E. Giusti, Global $C^{1, \alpha}$-regularity for second order quasilinear elliptic equations in divergence form, J. Reine Angew. Math. 351 (1984), 55-65. MR.749677 (85k:35077)

[13] David Gilbarg and Neil S. Trudinger, Elliptic partial differential equations of second order, Grundlehren der Mathematischen Wissenschaften, vol. 224, Springer-Verlag, Berlin, 1977. MR0473443 (57 \#13109)

[14] Pedro González-Rodríguez and Arnold D. Kim, Reflectance optical tomography in epithelial tissues, Inverse Problems 25 (2009), no. 1, 015001, 24, DOI 10.1088/0266-5611/25/1/015001. MR2465333(2010d:92069)

[15] Damon Hyde, Misha Kilmer, Dana H. Brooks, and Eric Miller, Analysis and exploitation of matrix structure arising in linearized optical tomographic imaging, SIAM J. Matrix Anal. Appl. 29 (2007), no. 4, 1065-1082, DOI 10.1137/060657285. MR2369284 (2008m:65096)

[16] Kimberly Kilgore, Shari Moskow, and John C. Schotland, Inverse Born series for diffuse waves, Imaging microstructures, Contemp. Math., vol. 494, Amer. Math. Soc., Providence, RI, 2009, pp. 113-122, DOI 10.1090/conm/494/09646. MR2581769(2011d:35517)

[17] Olga A. Ladyzhenskaya and Nina N. Ural'tseva, Linear and quasilinear elliptic equations, translated from the Russian by Scripta Technica, Inc., translation editor: Leon Ehrenpreis, Academic Press, New York, 1968. MR0244627 (39 \#5941)

[18] Gary M. Lieberman, Boundary regularity for solutions of degenerate elliptic equations, Nonlinear Anal. 12 (1988), no. 11, 1203-1219, DOI 10.1016/0362-546X(88)90053-3. MR.969499 (90a:35098)

[19] Gary M. Lieberman, The natural generalization of the natural conditions of Ladyzhenskaya and Ural'tseva for elliptic equations, Comm. Partial Differential Equations 16 (1991), no. 2-3, 311-361, DOI 10.1080/03605309108820761. MR1104103 (92c:35041)

[20] Nguyen Hoang Loc and Klaus Schmitt, On positive solutions of quasilinear elliptic equations, Differential Integral Equations 22 (2009), no. 9-10, 829-842. MR2553058 (2010k:35199)

[21] V. A. Markel and J. C. Schotland, Symmetries, inversion formulas, and image reconstruction for optical tomography, Phys. Rev. E 70 (2004), 056616.

[22] Shari Moskow and John C. Schotland, Convergence and stability of the inverse scattering series for diffuse waves, Inverse Problems 24 (2008), no. 6, 065005, 16, DOI 10.1088/02665611/24/6/065005. MR2456952(2010b:35488)

[23] Murray H. Protter and Hans F. Weinberger, Maximum principles in differential equations, Prentice-Hall Inc., Englewood Cliffs, N.J., 1967. MR.0219861 (36 \#2935)

[24] M. C. W. van Rossum and Th. M. Nieuwenhuizen, Multiple scattering of classical waves: microscopy, mesoscopy, and diffusion, Rev. Modern Phys. 71 (1999), 313-371.

[25] Leonid Ryzhik, George Papanicolaou, and Joseph B. Keller, Transport equations for elastic and other waves in random media, Wave Motion 24 (1996), no. 4, 327-370, DOI 10.1016/S0165-2125(96)00021-2. MR 1427483(98k:35199)

[26] J. C. Schotland, Direct reconstruction methods in optical tomography, Lecture Notes in Math., vol. 2035, Springer, Heidelberg, 2012, pp. 1-29. MR3024668

[27] J. C. Schotland and V. A. Markel, Inverse scattering with diffusing waves, J. Opt. Soc. Amer. A 18 (2001), 2767-2777.

[28] L. V. Wang, Mechanisms of ultrasonic modulation of multiply scattered coherent light: an analytic model, Phys. Rev. Lett. 87 (2001), 043903. 
Department of Mathematics and Applications, Ecole Normale Supérieure, 45 Rue D'Ulm, 75005 PARIS, France

E-mail address: habib.ammari@ens.fr

Institut Langevin, ESPCI Paristech, CNRS UMR 7587, 10 Rue Vauquelin, 75231 Paris Cedex 05, France

E-mail address: emmanuel.bossy@espci.fr

Laboratoire de Probabilités et Modèles Aléatoires \& Laboratoire Jacques-Louis Lions, Université Paris VII, 75205 Paris Cedex 13, France

E-mail address: garnier@math.jussieu.fr

Department of Mathematics and Applications, Ecole Normale Supérieure, 45 Rue D'Ulm, 75005 PARIS, France

E-mail address: Inguyen@dma.ens.fr

Department of Mathematics and Applications, Ecole Normale Supérieure, 45 Rue D'Ulm, 75005 PARIS, France

E-mail address: laurent.seppecher@ens.fr 